\title{
Linked Services for Enabling Interoperability in the Sensing Enterprise
}

\author{
Matthias Thoma ${ }^{1,2}$, Alexandru-Florian Antonescu ${ }^{1,2}$, \\ Theano Mintsi ${ }^{1}$, and Torsten Braun ${ }^{2}$ \\ 1 SAP (Switzerland) Inc. \\ SAP Research Zurich \\ Althardstrasse 80, 8105 Regensdorf, Switzerland \\ 2 University of Bern, \\ Institute of Computer Science and Applied Mathematics \\ Communication and Distributed Systems Research Group \\ Neubrueckstrasse 10, Bern, Switzerland
}

\begin{abstract}
In future, the so called "sensing enterprise", as part of the Future Internet, will play a crucial role in the success or the failure of an enterprise. We present our vision of an enterprise interacting with the physical world based on a retail scenario. One of the main challenges is the interoperability not only between the enterprise IT systems themselves, but also between these systems and the sensing devices. We will argue that semantically enriched service descriptions, the so called linked services will ease interoperability between two or more enterprises IT systems, and between enterprise systems and the physical environment.
\end{abstract}

Keywords: Linked services, enterprise integration, wireless sensor network, smart items, retail, sensing enterprise.

\section{Introduction}

One of the main challenges of future enterprise IT systems is the usage of data collected from the real world in real time, contextualizing it and providing the user of these systems with the best possible up to date information to base business decisions on. We will call the vision of context-aware and real-world aware enterprise IT systems the "Sensing Enterprise".

The emerging sensing enterprise makes cooperation and interoperability between enterprises, and more specifically, between heterogeneous enterprise IT systems on one hand more important than already today, but on the other hand also more complicated. It is a well known problem, that enterprises today have a need for collaboration, but because of interoperability issues between enterprise IT systems this is often not as successful as it would be desirable [1].

Traditionally enterprise IT vendors base their system on service oriented architectures (SOA). Furthermore they mainly use high-level, often proprietary, protocols. Unfortunately, this imposes a specific problem when implementing the sensing enterprise. The integration of sensing devices, which deliver the context information needed on the state of the physical world (like mobile phones, 
sensor networks, RFID readers and tags), is still performed manually and does not integrate well into SOA architectures of nowadays IT landscapes.

A recent approach to describe services in a SOA environment are semantically enriched service descriptions, based on RDF and semantic web technologies. In this paper we will present our vision of the sensing enterprise based on these next generation Linked Services. We use them to access sensor devices, which are able to describe themselves, thus enabling a sensing enterprise which seamlessly integrates into today's enterprise world. Furthermore, we will discuss an integration middleware which can be used to connect ERP systems to the physical world. We conclude by presenting a case study of applying the integration middleware, a reasoning system and data analysis to a real-world scenario.

\section{Related Work}

The idea of using Linked Data for service descriptions has received wider attention recently. There are approaches known as linked service (e. g. 2], [3] and [4]), which contribute to the web of data by applying ontologies for service descriptions and discovery. iServe [5], for example, aims to support service publishing and discovery in a better way. Service descriptions, on the other hand, have attracted a lot of attention in the context of the Internet of services. The most well known standard is the WS-* family, which centers around the Webservice Description language (WSDL) [6].

Many existing service description languages focus more or less on the description of technical interfaces, sometimes annotated with quality parameters. We currently use an RDF-based version of USDL (Unified Service Description Language), which addresses — in addition to the technical aspects — businessrelated properties, capabilities and non-functional characteristics [7].

The main difference between our approach and ontology-based linked service approaches, which try to contribute to the web of data, is that we focus purely on services, which do not necessarily have to be part of the web of data. This includes their capabilities and their integration into current enterprise IT systems.

The general problem of integrating smart items and data gathered from sensor networks is well known in the literature. Gomez et. al. [8] propose an additional layer called Enterprise Integration Component (EIC), which is a generic mediation layer between enterprise systems and the WSN middleware.

Two approaches which are also addressing some business aspects are from Glombitza et. al. 9] and from Carracas [10. Glombitza et. al. propose the usage of standard web service technologies (SOAP, XML). They also target for using SOA principles aiming towards the integration with BPEL, nonetheless their approach is based on WSDL and covers only the pure technical aspects of SOA. Carracas [10] concentrates on WSN integration via BPMN and compiling corresponding code for running it on the mote.

Sensor description languages are used to model the characteristics, as well as the input and output parameters of sensing services. SensorML [11], for example, 
is an XML based modelling language, which allows specifying each sensor by its meta-data. It allows to model processes that are linked together through inputs and outputs.

\section{$3 \quad$ Terminology}

In this section we will properly define some terms we will use throughout this paper. Furthermore we provide some brief background informations on technologies used as this paper is combining ideas from different research areas (e. g. linked services, semantic web, business networks, enterprise systems, smart items, wireless sensor networks) and not ever reader might be fluent in all of these fields.

Enterprise System. We use the term enterprise system (often called ERP or Enterprise Resource planning system) as follows [8]: An ERP is a set of business applications that allows large enterprises to run all phases of an enterprise's operations to facilitate cooperation and coordination of work across the enterprise. The ideal enterprise system could control all major business processes in real time. Enterprise systems have in general high requirements concerning availability, scalability, reliability as well as security and interoperability.

Wireless Sensor Network. A wireless sensor network (WSN) is a network consisting of wirelessly connected small embedded devices (motes). The devices are equipped with one or more sensors, a microcontroller, radio transceiver, some memory and a power supply. Sensor nodes are often battery powered. Usually they are very restricted devices to reduce the cost per unit. They allow an enterprise to perceive the state of its environment in real-time at a low cost.

Interoperability. There has been a lot of research on enterprise interoperability in recent years. The term interoperability is defined by IEEE as "the ability of two or more systems or components to exchange information and to use the information that has been changed." [12. More information on the theory behind enterprise interoperability can be found, for example, in [1] and [13].

OLTP (On Line Transaction Processing) and OLAP (On Line Analytic Processing) are two complementing technologies, which are used to facilitate business processes and enable business intelligence respectively. While OLTP focuses on serving the maximum possible number of short transactions as fast as possible and on maintaining vast, multi-access warehouses consistent, OLAP concentrates on complex, low-volume transactions over multi-dimensional data in order to support decision support and problem solving.

$R D F$ and $S P A R Q L$. In search of a general method for interchanging data over the Web, W3C introduced Resource Description Framework (RDF). This simple semantic data model uses URIs to name things and the relationships between them, creating this way triples which can be conceptualized as directed, labeled graphs. RDF enables the easier integration of data in the web, an extension of existing schemas and a data consumption through the use of SPARQL queries. A more in-detail presentation can be found in 14 . 


\section{Sensing Enterprise}

The sensing enterprise is a catch phrase for the ongoing integration of physical data captured by sensors into ERP systems and the on-demand utilization (sometimes refereed to as sense making) of this data. This will allow a tight integration of events and processes in the physical world to be used in business processes. In the following two sections we will first present the key drivers of our current research, and then go on with explaining how semantically enriched linked services help solving the problems we identified.

\subsection{Key Drivers}

We see three key drivers in the sensing enterprise context, which motivates our current research. In section 5.1 we will show that the linked service paradigm is an ideal candidate for fulfilling these requirements. The three key drivers are:

Interoperability. In the past (and even nowadays, but at a lower level) ERP vendors used to base their system on proprietary protocols. Interoperability, as a result, meant implementing custom connectors to these services. To some degree this was caused by historical, technical reasons and the lack of agreed-on standards. Nowadays, in the days of coopetition interoperability has become more important. Thinking further in the future, especially in the sensing enterprise, where we might deal with all kinds of smart items from several vendors, which have to integrate into various backend systems, flexible and smart interoperability is a must. Furthermore, even nowadays, when we talk about enterprise interoperability there is specific knowledge of the used protocol and the data necessary. Future Enterprise systems need to target interoperability at a semantic level as well.

Enablement of Sense-Making. Future Enterprise backend systems will have to do reasoning on data coming from various sources. The description of these services should therefore allow semantic annotations, which are to be understood and processed by enterprise IT systems. As we will describe later, we are following a very pragmatic approach here, without the need of being fully reasonable in a theoretical sense.

Enablement of Real-Time Business Decision Support. In many industries the backend systems are still rather disconnected from what is actually happening. Often, the information is gathered a-posteriori and deviations from the planned state are detected late. Integrating real-time decision support into these systems will enable a business to run more efficient, react timely on changes in the business process and allow a proper exception handling. While this closely related to sense-making, real-time business decision support does not only rely on sense-making, it would be even possible without semantic (ontological) reasoning by utilizing domain knowledge and complex event processing, as it is already done today. 
There are two recent emerging technologies which enables the sensing enterprise become a reality:

Real Time Big Data Analytics. A typical enterprise generates very large and diverse data sets coming from its distributed business locations. Besides OLAP data, the enterprise might also record data produced in social networks, surveillance devices or by third party systems owned by business partners. These massive amounts of detailed data can be combined and analyzed by predictive analytics, data mining or statistics. Doing this process in real-time, for example, by using in-memory data processing, creates a business advantage for the company by giving insight into the real-world dynamics of their business.

Sensor Networks and Near Field Communication. Sensor networks are starting to complement the already existing RFID (Auto ID) technologies that are already available on the market.

\subsection{The Sensing Enterprise from a Retailer's Point of View}

As the authors have the most proficient background in retail, we will illustrate the application and usefulness of the above-mentioned concepts in the retail domain. We will therefore just have a brief look on the transportation of goods from the producer to the retailer and, of course, finally to the consumer. Furthermore, we will focus on the data that can be gathered in the stores. An exemplary integration into enterprise IT systems is then discussed in section 6 .

The spirit of the sensing enterprise will be everywhere in the stores of the future. In the retail store and in the supporting supply chain physical and social realities change at a high pace. In the stores themselves this includes different exceptional situations, such as out-of-shelve situations, technical problems or congestions at the point of sell. As an example, monitoring the freeing systems would help to prevent loss. If one freezing machine failed then the ERP would suggest shuffling them to other freezers and/or starting immediate discounts. In such a case the discount could be determined by checking the product validity ranges (e.g. temperature) associated with a product description, located in the manufacturer ERP system. The store's ERP would combine the sensor data with the remote descriptions in order to trigger the discount decision.

Furthermore, there are a magnitude of forecasts possible to avoid out-of-shelve situations or to detect unexpected high or low demand and to react on real-time, including sending the personnel to the right spot or to suggest changes in the product placement. This of course, does not apply to a single store only. Most stores are part of enormous, sometimes multi-national corporations, where predictions and forecasts based on information gathered from all stores contribute to one huge knowledge base.

An important and competitive requirement in the retail world is to deliver products according to a predefined agreement. A Service Level Agreement (SLA) defines the conditions under which a product is expected to get transported and delivered to the stores. Sensing comes into play to detect the conditions, that 
when deviating from the SLA, cause an internal system change and demand or even bring changes in the whole retail process. Through enriching this part of the retail procedure with sensing technologies and SLAs, every involved enterprise can benefit by reducing risks, saving time, reducing cost and increasing sales and earnings.

In our scenario, a sensor-equipped "intelligent container" is responsible for monitoring the goods. The monitoring process is driven by a device-constrained SLA, which would determine which sensors are used and how the information is processed. Upon arriving at a distribution point, such as a harbor or an airport, edge sensors of the transporter share their SLAs with the ERP of the distributor, while they also communicate their collected information to the central enterprise system of the receiving retailer. As the sensors are resource-constrained, the SLAs are not actually stored on them, but only a link is provided to the complete SLA description which resides in the transporter's ERP. An extra benefit is that this procedure creates an accurate track of the products transportation in terms of localization and transportation conditions. The entire tracking history would be recorded as ERP transactions in the intermediary ERP systems. Such information can prove vital to the retailer in terms of tracking back problems, deciding on its collaborators and defining the transportation costs.

At the next stage of the supply chain goods are at the selling point available for the customers. Following the same logic as in the transportation phase, there are wireless sensors on the shelves measuring all crucial parameters, carrying the SLAs and sending collected data to edge sensors, which in turn forward them to the store ERP. Upon arriving at the store, the SLA of every product is loaded on the local ERP, so that it is aware of the characteristics and maintenance conditions of each of them.

Sensing the conditions, under which products are maintained, and making decisions on the sensor level facilitates and expedites finding a solution. Analyzing the sensed data at real-time serves the retailers as a useful source of information for further investments and re-arranging the supply chain. In the retail world all that translates to time, successful collaborations, building a strong brand name and earnings.

Moving from the actual store, its supply chain and personnel to the consumers: The sensing enterprise will enable new ways of interacting with the consumers. Interactions with the consumer via mobile devices are then possible based on the consumers context. The smartphone use would also help track the user and then combining data from in-store video cameras and location processing would produce some dynamic price changes or voucher generation which would be then sent to the buyers smartphones, published on store's website, advertised in store, etc.

\section{$5 \quad$ Linked Services and the Sensing Enterprise}

As part of an enterprise level SOA platform, service descriptions are used to describe the functional and non-functional properties of a service, including but 
not limited to its technical interface. We follow the idea of using linked services which we will describe in detail in section 5.1 and then go on showing how they can be used in a sensing enterprise context in section 5.2 . In this context we will present an enterprise architecture, which allows interacting with smart items based on linked services.

When talking about service descriptions it is important to distinguish between the actual deployed (and running) service and the description of the service. A linked service therefore describes a technical or non-technical service, it is not the service itself and thus does not have, for example, a technical service endpoint. It can describe one though, if the service described has one. A thorough classification and taxonomy of services and the difference to a service description can be found in [15]. Furthermore, as service is a term which can have many meanings. One wide-spread use of service is, especially in computer science, to use it as a synonym for a technical interface. We are following the approach of Barros et. al., where in [16] a service is defined as a "commercial transaction where one party grants temporary access to the resources of another party (...)".

A more thorough discussion of linked services can be found in [17] and [5]. In this work we do not dive deep into the realm of service science. We present enough background information for the reader to understand our contribution, but we limit ourselves to the sensing enterprise and more specifically the points were linked services contribute to that vision. A good and comprehensive overview can be found in [16].

\subsection{Linked Services}

In a nutshell the idea of linked services is to base service descriptions on standard technologies known from the semantic web (e. g. Ontologies, OWL and RDF). Furthermore, these semantically enriched service descriptions are following the linked data idea as outlined by Berners-Lee [18. He suggested the following four simple rules for publishing data on the web, thus creating one single data space - the web of data:

1. Use URIs as names for things

2. Use HTTP so that people can look up those names

3. When someone looks up a URI, provide useful information, using the standards (RDF, SPARQL)

4. Include links to other URIs, so that they can discover more things

Compared to the very ambitious idea of the web of data our vision is way more focused on the interoperability between enterprise systems, and enterprise systems and end-user devices. We currently see reasoning applicable on a domain level only. In contrast to many research as it is going on today in the semantic web, we do not want to model or understand the whole world. Our mid-term goal 
is semantic interoperability in very specific domains (for example in retail). For this, we foresee the use of light-weight ontologies. Furthermore, we would even allow manual steps, like hard-coded rules by domain experts, in the processing of this services. Research on interoperability has shown the need of semantic interoperability, this is sometimes complemented by the need of pragmatic interoperability. Our approach does not solve all the problems that can arise from wrong assumptions on either the semantic or the pragmatic level, but the use of semantic technologies and the restriction on a domain-level should reduce the risk of making wrong assumptions on both sides of the communication channel.

Linked Services do not apply only to the description of one single service provided by one service provider. Linking services together will allow an even tighter integration of business partners, thus contributing to the idea of service networks [16].

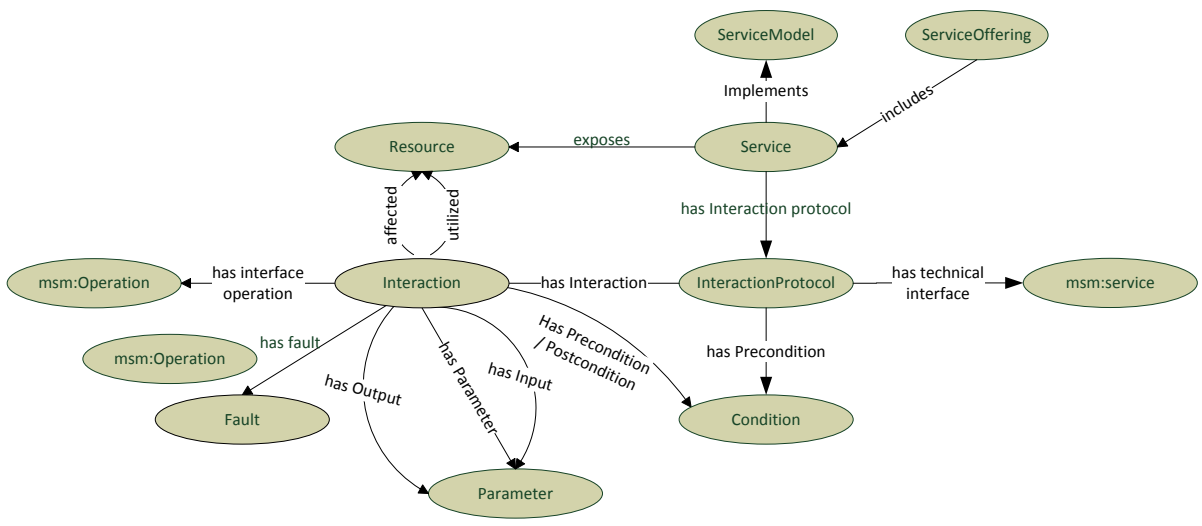

Fig. 1. Excerpt from the Linked USDL ontology

We use a specific implementation of Linked Services for expressing functional and non-functional properties, namely Linked USDL [2. To maximize interoperability Linked USDL adopts, whenever possible, existing RDF(S) vocabularies such as the Minimal Service Model and FOAF. Linked USDLs objective is to develop an ontology to represent services. It therefore creates explicit ontological links to domain specific ontologies. While this is the origin of the name linked services, the authors see a lot of potential also in actually linking one part of the service description to more detailed information defining the very same service. An excerpt of the Linked USDL ontology is shown in figure 1.

To our knowledge Linked USDL is the only standardization effort driven by large corporations with the goal of expressing not only purely functional aspects of a service, but also the business and operational aspects. A comprehensive introduction into each can be found in [16]. 
A very concrete service description for a smart item, in this case a simple temperature sensor as it could be used in a shops freezers, looks as follows:

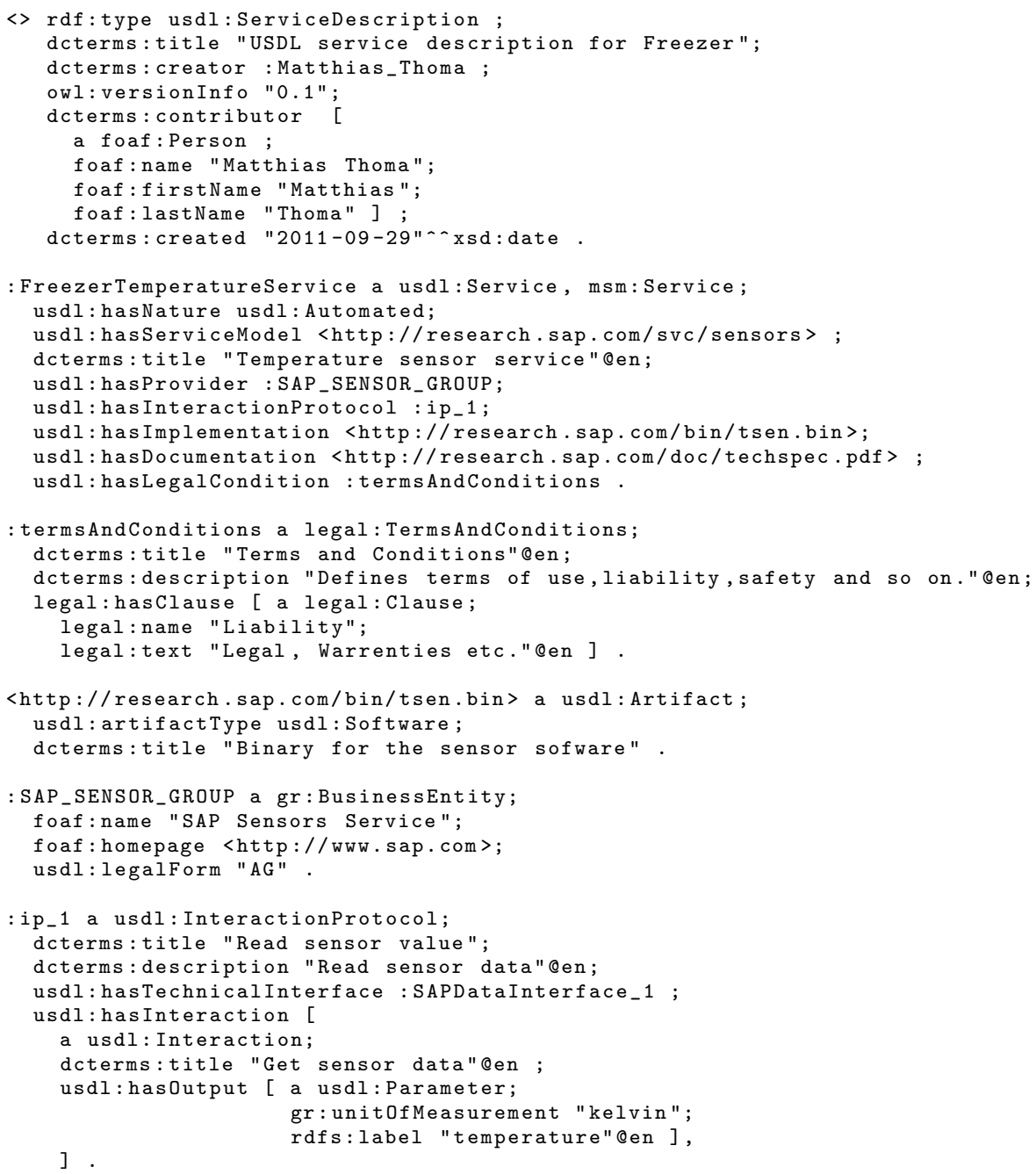

In this simple example one can already see the advantages of Linked Services. The interaction protocol describes how to access the service in a technical way and provides semantics on the parameters. These can be used in a reasoning engine of an ERP system. The whole service description is based upon standard technologies. It can be queried using SPARQL and information that can be found elsewhere can be directly linked to the service description. There is no need for any additional layer.

Linked services is a very powerful concept, which goes way beyond our usage as an ERP interoperability and service exchange platform. For example, there already exists an marketplace for tradeable services, service repositories and registries based on Linked USDL. 


\subsection{Extending Linked Services to Sensors and Smart Items}

In this section we will provide an overview of how linked services can be used to enable the sensing enterprise. We will present the architecture of a nextgeneration linked services enterprise platform and describe how we apply lightweight service descriptions to smart items and sensor networks. We are not going into too many details on how linked services integration is done in regular enterprise networks and service marketplaces, but present our extension to the concept. For more information on that we need refer to the already existing literature (e. g. [17] or [5]).

As shown in [19] a basic, shrunk to the essentials, version of the service description on the smart item which links to other parts of the service description (like comprehensive SLAs) is enough to enable interactions with sensor motes. Our platform, as shown in Fig. 1 enables an enterprise IT system to access and evaluate linked services.

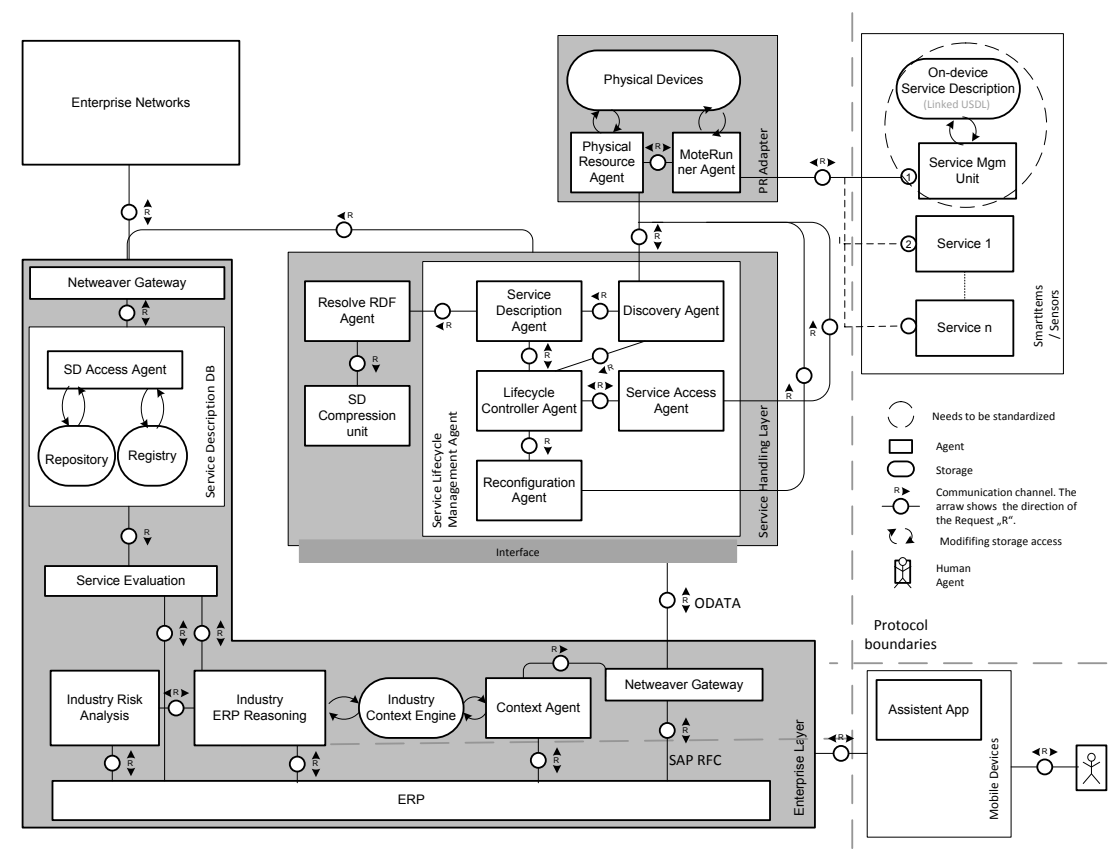

Fig. 2. High level block diagram of the linked service enterprise platform. (We are following the TAM standard for modeling the architecture)

As we consider smart items (sensor motes) as first-class entities in our SOA architecture, they are able to describe themselves through linked services descriptions. The actual services are accessed via the service management unit. Interoperability is achieved via a standardization of the interface to the management unit and of the service description language. Currently, CoAP seems to be a good candidate in addition to linked services. This allows different ERP vendors 
to implement their own integration platform without loosing interoperability. These self-description capabilities allow the smart items joining the realm of an ERP backend system to automatically integrate into the system based on the semantic information found in the service description, trigger backend actions and access the services on the smart item.

The Service Handling Layer is taking care of the actual handling of services on smart devices. It is exposed to the Enterprise Layer through a SOA compatible interface and is thus integrating into an enterprise' SOA environment. Within the Service Handling Layer there are several agents which we will describe briefly: The Discovery Agent (DA) is interacting with the Physical Resource Adapter (PRA) and discovers new services available on new smart items. Communication with and monitoring of smart items is done by the PRA. New service descriptions are first completed by the Resolve RDF Agent and (in case of compression) uncompressed by the Service Description Compression unit. The Service Description agent is then responsible for adding the service to the ERPs service registry and repository, thus making it available to the enterprise.

Having a closer look into the enterprise layer, the service descriptions are exposed to other ERP systems via a gateway using a standardized protocol. In addition to what already exists today in business intelligence (e. g. demand forecasting, campaing management, price calculations), we make use of context acquired by sensors. Based on this information, combined with historical data it is possible to do reasoning and real-time risk analysis. We currently see the short-term to mid-term applicability of this approach at a domain-specific or industry specific level.

The communication between the enterprise backend system and the SOA integration platform is commonly done via a gateway component (for example SAP Netweaver Gateway separating the ERP and auxiliary services. As the integration platform is for the ERP system also just a service, communication is done through a standard interface known to the enterprise SOA environment. Nowadays, ERP systems still use a lot of heavy-weight proprietary protocols, e. g. BAPI or SAP RFC, while the communication with the SOA platform can be done via a standardized protocol. The industry is currently moving towards the Open Data Protocol (OData) [20].

\section{Applying Linked Services to the Sensing Enterprise}

In this section we will present how our integration platform as described in the previous section and the concept of linked services enable realizing the sensing retailer as presented in section 4.2. We will assume that all enterprises involved are already linked services enabled.

Looking at the ERP systems of collaborating enterprises or cooperating ERPs withing one enterprise, there are usually many service invocations involved. They grant each other (restricted) access to their service repositories. The fact that they are semantically enriched allows reasoning even between systems. The retail sector is an ideal candidate for being used as pioneering industry, as there are 
already exchange formats (e. g. EDI) and identification systems for goods (e. g. EPCglobal) available. Furthermore, the retail industry already uses some sensing technologies like RFID tags and AutoID quite heavily. On the business side, existing business analytics are already working with these information, which allows incremental, non-disruptive, innovations to be performed.

We foresee a wide-spread use of sensing technologies used in the retail industry, e. g. within the supply chain as intelligent containers, for tracking of goods and their conditions or for tracking customers and personnel. Thanks to the selfdescription capabilities of these smart items the ERP system can communicate with them and exchange, for example, the SLAs and the corresponding sensed condition of goods.

In the following we will present a SLA document, which could be used for monitoring the temperature of goods. The TransportationProfile would then be referred from the sensor as residing in a remote ERP system.

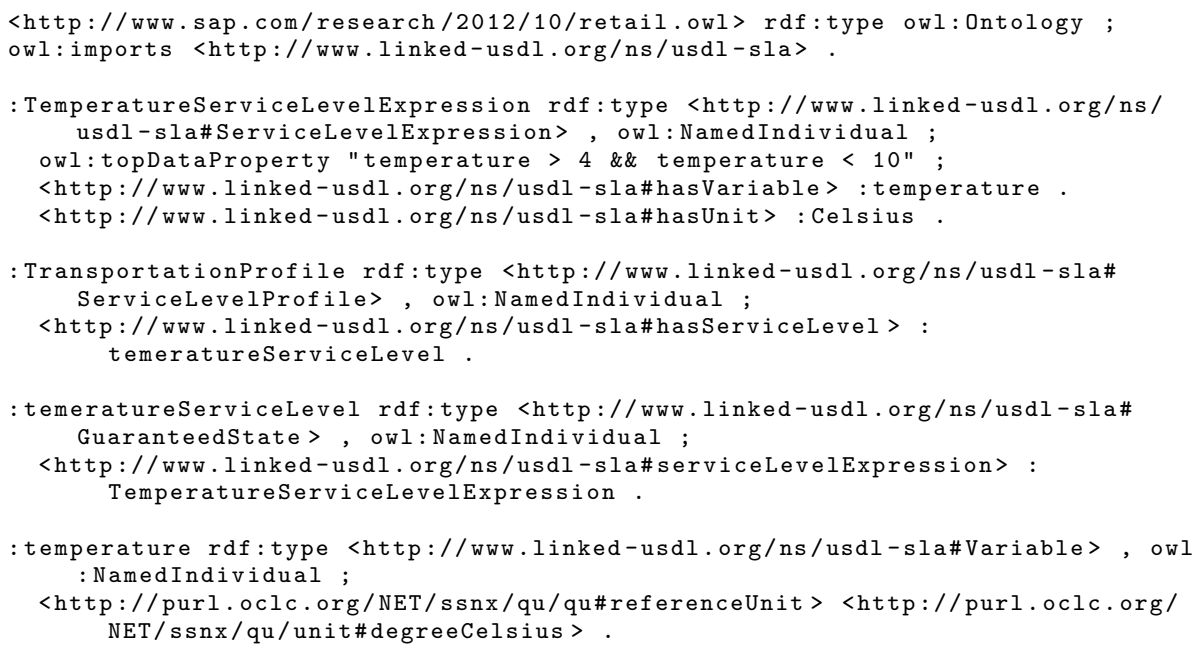

The temperate here would have to be within an temperate range of $[4,10]$ degrees Celsius.

Within business analytics there are already many solutions available which work with near-realtime information. This can be transformed to real-time by leveraging big data technologies. The granularity of the results can be increased by using sensing technologies. Exception management, today a manual tasks as the ERP systems are often unaware of the real conditions the goods are in, will become easier. The store itself collects data about goods and customers, contextuilzes it and thus improves the business analytics process. Furthermore, a direct interaction with personel (e. g. sending them directly to out-of-shelve situations, or to supreme cusomers) and customers will be possible. 


\section{Further Work}

In this paper we focused on the interoperability part and the vision of semanticaly enriched service descriptions for business intelligence systems. We will continue our work and integrate mobile devices also as first class entities in our platform and utilize data gathered from them. Furthermore, we are working on extending real systems, which are already using some kind of structured data, to use semantic technologies and to improve the quality of the results by using context information.

\section{Conclusion}

In this paper we extended the scope of Linked Services to the Sensing Enterprise. We specifically addressed the integration of semantically enriched Service Descriptions, which additionally follow the Linked Data principles, and of real-time sensed data into distributed Enterprise Systems and the involved business processes. The final goal is on one hand to enable decision-making at the low-level embedded devices supporting business processes and reduce thereby the time response in critical situations, while on the other hand to allow both low-level devices and backend of different enterprises to communicate independently and efficiently empowering the inter- operation of the involved enterprises. We presented all necessary concepts and the way we make use and elaborate on them, a motivating scenario in the retail world, whereas we also illustrated our suggested integration platform for applying the Linked Services in the Sensing Enterprise.

Acknowledgment. This research received funding from the European Commission under grant 257521 (IoT-A) and grant 285248 (FIWARE). The authors want to thank the Mote Runner team at IBM Research for their support.

\section{References}

1. van Sinderen, M.: Challenges and solutions in enterprise computing. Enterprise Information Systems 2(4), 341-346 (2008)

2. Leidig, T., Pedrinaci, C.: Linked usdl (2012)

3. Dietze, S., Yu, H.Q., Pedrinaci, C., Liu, D., Domingue, J.: SmartLink: A Web-Based Editor and Search Environment for Linked Services. In: Antoniou, G., Grobelnik, M., Simperl, E., Parsia, B., Plexousakis, D., De Leenheer, P., Pan, J. (eds.) ESWC 2011, Part II. LNCS, vol. 6644, pp. 436-440. Springer, Heidelberg (2011)

4. Speiser, S., Harth, A.: Taking the lids off data silos. In: Proceedings of the 6th International Conference on Semantic Systems, I-SEMANTICS 2010, pp. 44:144:4. ACM, New York (2010)

5. Pedrinaci, C., Liu, D., Maleshkova, M., Lambert, D., Kopecky, J., Domingue, J.: iServe: a linked services publishing platform. In: Workshop on Ontology Repositories and Editors for the Semantic Web, ORES 2010 (2010)

6. Christensen, E., Curbera, F., Meredith, G., Weerawarana, S.: Web services description language (wsdl). Technical report (March) 
7. Charfi, A., Schmeling, B., Novelli, F., Witteborg, H., Kylau, U.: An overview of the unified service description language. In: 2010 IEEE 8th European Conference on Web Services, ECOWS (December 2010)

8. Gomez, L., Laube, A., Sorniotti, A.: Design guidelines for integration of wireless sensor networks with enterprise systems. In: Proceedings of the 1st International Conference on MOBILe Wireless MiddleWARE, Operating Systems, and Applications, MOBILWARE 2008, pp. 12:1-12:7. ICST, Brussels (2007)

9. Glombitza, N., Pfisterer, D., Fischer, S.: Integrating wireless sensor networks into web service-based business processes. In: Proceedings of the 4th International Workshop on Middleware Tools, Services and Run-Time Support for Sensor Networks, MidSens 2009, pp. 25-30. ACM, New York (2009)

10. Caracaş, A., Kramp, T.: On the Expressiveness of BPMN for Modeling Wireless Sensor Networks Applications. In: Dijkman, R., Hofstetter, J., Koehler, J. (eds.) BPMN 2011. LNBIP, vol. 95, pp. 16-30. Springer, Heidelberg (2011)

11. Open Geospatial Consortium. Technical report

12. Institute of Electrical and Electronics Engineers: IEEE standard computer dictionary: A compilation of IEEE standard computer glossaries, New York, NY (1990)

13. Chen, D., Doumeingts, G., Vernadat, F.: Architectures for enterprise integration and interoperability: Past, present and future. Computers in Industry 59(7), 647659 (2008); Enterprise Integration and Interoperability in Manufacturing Systems

14. Klyne, G., Carroll, J.J., McBride, B.: Resource Description Framework (RDF): Concepts and Abstract Syntax. W3C Recommendation, W3C (February 2004)

15. Thoma, M., Meyer, S., Sperner, K., Meissner, S., Braun, T.: On iot-services: Survey, classification and enterprise integration. In: 2012 International Conference on and 5th International Conference on Cyber, Physical and Social Computing Internet of Things, iThings/CPSCom (November 2012)

16. Barros, A., Oberle, D.: Handbook of service description USDL and its methods. Springer, New York (2012)

17. Pedrinaci, C., Domingue, J.: Toward the next wave of services: Linked services for the web of data 16(3) (2010)

18. Berners-Lee, T.: Linked data - design issues (2006)

19. Thoma, M., Sperner, K., Braun, T.: Service descriptions and linked data for integrating wsns into enterprise. In: 2012 Third International Workshop on Software Engineering for Sensor Network Applications, SESENA (June 2012)

20. OData Technical Committee: Open data protocol (2012) 